PROCEEDINGS OF THE

AMERICAN MATHEMATICAL SOCIETY

Volume 135, Number 5, May 2007, Pages 1279-1282

S 0002-9939(06)08633-3

Article electronically published on November 13, 2006

\title{
THE DEGREE OF THE BICANONICAL MAP OF A SURFACE WITH $p_{g}=0$
}

\author{
MARGARIDA MENDES LOPES AND RITA PARDINI
}

(Communicated by Michael Stillman)

\begin{abstract}
In this note it is shown that, given a smooth minimal complex surface of general type $S$ with $p_{g}(S)=0, K_{S}^{2}=3$, for which the bicanonical map $\varphi_{2 K}$ is a morphism, the degree of $\varphi_{2 K}$ is not 3. This completes our earlier results, showing that if $S$ is a minimal surface of general type with $p_{g}=0$, $K^{2} \geq 3$ such that $\left|2 K_{S}\right|$ is free, then the bicanonical map of $S$ can have degree 1,2 or 4 .
\end{abstract}

\section{INTRODUCTION}

Complex surfaces of general type with $p_{g}=0$ continue to be intriguing. Continuing our study of their bicanonical map, in this note we prove the following.

Theorem 1.1. Let $S$ be a smooth minimal complex surface of general type with $p_{g}(S)=0$ and $K_{S}^{2}=3$ such that the bicanonical system $\left|2 K_{S}\right|$ is base point free. Then the degree of the bicanonical map of $S$ is different from 3.

Theorem [1.1, together with previous results ([ML], [MP1], [MP2]) gives the following general statement on the degree of the bicanonical map of a surface of general type with $p_{g}=0$ and base point free bicanonical system.

Theorem 1.2. Let $S$ be a smooth minimal complex surface of general type with $p_{g}(S)=0$, and let $\varphi: S \rightarrow \mathbb{P}^{K^{2}}$ be the bicanonical map. Then:

(i) if $K_{S}^{2}=9$, then $\varphi$ is birational;

(ii) if $K_{S}^{2}=7,8$, then $\operatorname{deg} \varphi \leq 2$;

(iii) if $3 \leq K_{S}^{2} \leq 6$ and $\left|2 K_{S}\right|$ is base point free, then $\operatorname{deg} \varphi$ is equal to either 1,2 or 4 .

The proof of Theorem 1.1 is based on some properties of nonnormal rational quartic surfaces of $\mathbb{P}^{3}$, which are studied in the next section.

Notation and conventions. We work over the complex numbers; all varieties are assumed to be compact and algebraic.

We do not distinguish between line bundles and divisors on a smooth variety. Linear equivalence of divisors is denoted by $\equiv$ and numerical equivalence by $\sim$. The remaining notation is standard in algebraic geometry.

Received by the editors May 11, 2005 and, in revised form, December 16, 2005.

2000 Mathematics Subject Classification. Primary 14J29.

(C)2006 American Mathematical Society Reverts to public domain 28 years from publication 


\section{NonNORMAL RATIONAL QUARTICS OF $\mathbb{P}^{3}$}

In this section we show the following.

Theorem 2.1. Let $\Sigma \subset \mathbb{P}^{3}$ be a rational quartic surface such that $\Sigma$ is not normal, i.e. such that the singular locus of $\Sigma$ has dimension 1 . Let $\Upsilon \rightarrow \Sigma$ be the minimal desingularization and let $|H|$ be the pullback on $\Upsilon$ of the linear system of planes of $\mathbb{P}^{3}$. If the linear system $|H|$ is complete, then:

(i) the genus $g(H)$ of $H$ is equal to 2 ;

(ii) $K_{\Upsilon}^{2}=0$ and the linear system $\left|K_{\Upsilon}+H\right|$ is a free pencil of rational curves.

Proof. We start by noting that, since the linear system $|H|$ is the pullback on $\Upsilon$ of the linear system of planes of $\mathbb{P}^{3}$ and $\Sigma$ has degree $4, H^{2}=4$. The general curve $H$ of $|H|$ is smooth and irreducible by Bertini's theorem, and it is mapped birationally onto a singular plane quartic, thus we have $g(H)=1+\left(K_{\Upsilon}+H\right) H / 2 \leq 2$. On the other hand, by the regularity of $\Upsilon$ the restriction of $|H|$ to a general $H$ is a complete system, hence Riemann-Roch on $H$ gives $g(H) \geq 2$. This proves (i).

We divide the proof of assertion (ii) into steps. Consider the linear system $|D|:=\left|K_{\Upsilon}+H\right|$. By (i), we have $K_{\Upsilon} H=-2$ and thus $D H=2, D^{2}=K_{\Upsilon}^{2}$. Using the adjunction sequence for a general $H$ one sees that $h^{0}(D)=2$, namely $|D|$ is a pencil.

Write $|D|=F+|M|$, where $F$ is the fixed part of $|D|$ and $|M|$ is the moving part.

Step 1. $D$ is nef. In particular, we have $D^{2} \geq 0$. Since $q(\Upsilon)=0$, the restriction of $|D|$ to the general curve of $|H|$ is the complete canonical system. So for any irreducible component $\theta$ of $F$ we have $\theta H=0$ and, by the index theorem, $\theta^{2}<0$.

Let $\theta$ be an irreducible curve such that $\theta D<0$. Since $D$ is effective, $\theta$ is a component of $F$. Hence $\theta H=0, \theta K_{\Upsilon}<0, \theta^{2}<0$, namely $\theta$ is a -1-curve contracted by $|H|$, against the assumption that $\Upsilon \rightarrow \Sigma$ is the minimal desingularization.

Step 2. One has $D^{2} \leq 1$. If $D^{2}=1$, then $H=-2 K_{\Upsilon}$. The inequality $D^{2} \leq 1$ follows by the index theorem applied to $D$ and $H$. If $D^{2}=1$, the index theorem gives also $H \sim 2 D$, i.e. $H \sim-2 K_{\Upsilon}$. Since $\Upsilon$ is rational, we actually have $H \equiv$ $-2 K_{\Upsilon}$.

Step 3. The case $D^{2}=1$ does not occur. By Step 2 , if $D^{2}=1$, then $-K_{\Upsilon}$ is nef and $K_{\Upsilon}^{2}=1$. Riemann-Roch gives $h^{0}\left(-K_{\Upsilon}\right)=2$. Write $\left|-K_{\Upsilon}\right|=\Delta+|N|$, where $\Delta$ is the fixed part and $|N|$ is the moving part. We have

$$
1=K_{\Upsilon}^{2}=-K_{\Upsilon} \Delta-K_{\Upsilon} N \geq-K_{\Upsilon} N=N^{2}+\Delta N \geq 0
$$

Assume that $\Delta \neq 0$. Then, since a nef and big divisor is 1-connected, we have $\Delta N>0$. Hence in this case all the previous inequalities are equalities, and we have

$$
\Delta N=1, \quad N^{2}=0, \quad K_{\Upsilon} \Delta=0 .
$$

It follows that $\Delta^{2}=-K_{\Upsilon} \Delta-N \Delta=-1$, contradicting the fact that $\Delta^{2}+K_{\Upsilon} \Delta$ is even by the adjunction formula. So we conclude that $\Delta=0$. Since $K_{\Upsilon}^{2}=1$, it follows that the general curve of $\left|-K_{\Upsilon}\right|$ is smooth and irreducible of genus 1 . Since $\left(-K_{\Upsilon}\right) H=2$, the restriction of $|H|$ to the general curve of $\left|-K_{\Upsilon}\right|$ is not birational, contradicting the assumption that $|H|$ is the pullback of a very ample system via a birational morphism. Hence the case $D^{2}=1$ does not occur. 
Step 4. $D^{2}=0$ and $|D|$ is a pencil of rational curves. We have $D^{2}=K_{\Upsilon}^{2}=0$ by the previous steps. Since $D$ and $M$ are nef, we have

$$
0=D^{2} \geq D M=M^{2}+M F \geq M F \geq 0 .
$$

It follows that $M^{2}=M F=F^{2}=0$. Hence, by Zariski's lemma (see [BPV], Lemma (8.2), p. 90) there is a rational number $\alpha \geq 0$ such that $F \equiv \alpha M$ as $\mathbb{Q}$-divisors. One has: $2=-K_{\Upsilon} D=-K_{\Upsilon} M(1+\alpha)$. Since $-K_{\Upsilon} M=\frac{2}{1+\alpha}>0$, by the adjunction formula we must have $-K_{\Upsilon} M=2, \alpha=0$. Hence $F=0$, and $|D|$ is a free pencil of rational curves.

\section{The proofs of Theorem 1.2 And Theorem 1.1}

Proof of Theorem 1.1. Since $K_{S}^{2}=3, h^{0}\left(S, 2 K_{S}\right)=K_{S}^{2}+\chi\left(\mathcal{O}_{S}\right)=4$. Let $\varphi: S \rightarrow$ $\mathbb{P}^{3}$ be the bicanonical map and denote by $\Sigma \subset \mathbb{P}^{3}$ the image of $\varphi$, which is a surface by [Xi].

We argue by contradiction. So from now on we assume that $\operatorname{deg} \varphi=3$. Since $\left|2 K_{S}\right|$ is free, we have

$$
4 K_{S}^{2}=12=\operatorname{deg} \varphi \operatorname{deg} \Sigma=3 \operatorname{deg} \Sigma,
$$

namely $\Sigma$ is a quartic surface.

Let $\Upsilon \rightarrow \Sigma$ be the minimal desingularization. In principle, $\Upsilon$ is either a K3 surface or a ruled surface. Since $\Upsilon$ is birationally dominated by $S$ and $q(S)=0$ by the inequality $\chi\left(\mathcal{O}_{S}\right)>0$ for surfaces of general type, we have $p_{g}(\Upsilon)=q(\Upsilon)=0$, and so $\Upsilon$ is rational. We note also that, since $\Sigma$ is the image of $S$ by a complete linear system, the linear system $|H|$, as in the assumptions of Theorem 2.1] is complete.

We wish to show that $\Sigma$ is not normal. Assume by contradiction that $\Sigma$ is normal. Being a quartic hypersurface, $\Sigma$ is a Gorenstein surface with $K_{\Sigma}=0$ and $p_{g}(\Sigma)=1$, namely there exists a nonzero 2 -form $w$ on $\Sigma$ which is regular outside the singular locus of $\Sigma$, which is a finite set. The pullback $w^{\prime}$ of $w$ to $S$ is a nonzero 2 -form which is regular on the complement of the union of the -2-curves of $S$ and of a finite set of points. It is well known (cf. [St, Lemmas 1.8 and 1.11) that such a form $w^{\prime}$ is actually regular on $S$, but this contradicts the assumption $p_{g}(S)=0$. So $\Sigma$ is not normal, and by Theorem 2.1 we have $g(H)=2$ and $K_{\Upsilon} H=-2$.

Consider the linear system $\left|K_{\Upsilon}+H\right|$. By Theorem 2.1 this system defines a fibration $f: \Upsilon \rightarrow \mathbb{P}^{1}$ whose general fibre is a rational curve. Since $K_{\Upsilon}^{2}=0$ by Theorem 2.1, the fibration $f$ is not relatively minimal. Let $\theta$ be an irreducible -1-curve contained in a fibre of $f$. We have $\theta\left(K_{\Upsilon}+H\right)=0$, namely $\theta H=1$. So the image of $\theta$ in $\Sigma$ is a line $L$. If $L^{\prime}$ is the pullback of $L$ on $S$, then we have $3=L^{\prime} \psi^{*} H=L^{\prime}\left(2 K_{S}\right)$, a contradiction.

Proof of Theorem 1.2. Statement (i) and (ii) are the main result of [MP1].

So we consider the case $3 \leq K_{S}^{2} \leq 6$. We have $\operatorname{deg} \varphi \leq 4$, by [ML. Since $\left|2 K_{S}\right|$ is free by assumption, we have

$$
4 K_{S}^{2}=\operatorname{deg} \varphi \operatorname{deg} \Sigma .
$$

Hence we have to exclude the cases $K_{S}^{2}=6, \operatorname{deg} \varphi=3$ and $K_{S}^{2}=\operatorname{deg} \varphi=3$. The former does not occur by [MP2, Thm. 1.1], and the latter is excluded by Theorem 1.1 . 


\section{ACKNOWLEDGements}

The first author is a member of the Center for Mathematical Analysis, Geometry and Dynamical Systems, IST and was partially supported by FCT (Portugal) through program POCTI/FEDER and Project POCTI/MAT/44068/2002. The second author is a member of G.N.S.A.G.A. and was partially supported by P.R.I.N. 2002 and 2004 "Geometria sulle varietà' algebriche" of M.I.U.R.

\section{REFERENCES}

[BPV] W. Barth, C. Peters, A. Van de Ven, Compact complex surfaces, Ergebnisse der Mathematik und ihrer Grenzgebiete, 3. Folge, Band 4, Springer 1984. MR0749574 (86c:32026)

[ML] M. Mendes Lopes, The degree of the generators of the canonical ring of surfaces of general type with $p_{g}=0$, Arch. Math., 69 (1997), 435-440. MR1473098 (98j:14050)

[MP1] M. Mendes Lopes, R. Pardini, The bicanonical map of surfaces with $p_{g}=0$ and $K^{2} \geq 7$, Bull. London Math. Soc., 33 (2001), 265-274. MR.1817764 (2002a:14042)

[MP2] M. Mendes Lopes, R. Pardini, The classification of surfaces with $p_{g}=0, K^{2}=6$ and non birational bicanonical map, Math. Annalen, vol. 329, Number 3 (July 2004), 535-552. MR2127989 (2005m:14067)

[St] J. H. M. Steenbrink, Mixed Hodge structure on the vanishing cohomology, in "Real and Complex Singularities, Oslo 1976" (P. Holm ed.), Sijthoff \& Noordhoff, The Netherlands (1977), 525-564. MR0485870 (58:5670)

[Xi] G. Xiao, Finitude de l'application bicanonique des surfaces de type général, Bull. Soc. Math. France, 113 (1985), 23-51. MR0807825 (87a:14035)

Departamento de Matemática, Instituto Superior Técnico, Universidade Técnica de Lisboa, Av. Rovisco Pais, 1049-001 Lisboa, Portugal

E-mail address: mmlopes@math.ist.utl.pt

Dipartimento di Matematica, Università di Pisa, Largo B. Pontecorvo, 5, 56127 Pisa, ITALY

E-mail address: pardini@dm.unipi.it 